Background The Dutch College of General Practitioners (NHG) has produced almost 100 guidelines. Guideline adherence is on average $70-75 \%$, but varies between general practitioners and between guideline recommendations. A computerised decision support system (CDSS) could improve guideline adherence.

Objectives To describe the development and implementation of a CDSS (NHGDoc) for general practice in the Netherlands.

Methods NHGDoc is a collaborative effort between the NHG and ExpertDoc, the company that developed the CDSS. Relevant recommendations of eight NHG guidelines were translated into algorithms, generating specific alerts. All alerts were reviewed and authorised by guideline developers of the NHG. NHGDoc was integrated in two out of seven electronic health record systems used in Dutch general practice. Since 2008, users were regularly interviewed regarding their experiences with NHGDoc.

Results Integration of eight guidelines in NHGDoc generated alerts in approximately $30 \%$ of all patient records. Alert frequency varied in accordance with disease prevalence. Currently, 1100 general practices (appr. 25\% of Dutch general practices) are using NHGDoc. Users highly appreciated the content and lay out of the alerts, but preferred more control regarding the timing and frequency of the alerts.

Discussion NHGDoc is well received and appreciated in general practice. To enhance the success of NHGDoc the user-friendliness of the system could be further improved. Future research should provide evidence on the effectiveness of NHGDoc in terms of improving quality of care.

Implications for Guideline Developers To improve guideline adherence, CDSSs should have user-friendly designs, including options to personalise the decision support to meet the needs of individual users.

\section{PLUGGED-IN (PROVIDING LIKEABLE AND UNDERSTANDABLE GUIDELINES USING GRADE IN THE EMR WITH DIRECT LINKS TO INDIVIDUAL PATIENT DATA) PHASE 2}

${ }^{1} \mathrm{~L}$ Brandt, ${ }^{2} \mathrm{~T}$ Elde, ${ }^{3} \mathrm{~T}$ Agoritsas, ${ }^{3} \mathrm{G}$ Guyatt, ${ }^{1} \mathrm{~A}$ Kristiansen, ${ }^{4} \mathrm{P}$ Alonso-Coello, ${ }^{5} \mathrm{E}$ Akl, ${ }^{6} \mathrm{~J}$ Meerpohl, ${ }^{1} \mathrm{P}$ Vandvik. ${ }^{1}$ Department of Medicine, Innlandet Hospital Trust, Gjovik, Norway; ${ }^{2}$ Health Informatics, Norwegian University of Science and Technology (NTNU), Trondheim, Norway; ${ }^{3}$ Department of Clinical Epidemiology and Biostatistics, McMaster University, Hamilton, Canada; ${ }^{4}$ Iberoamerican Cochrane Centre, Institute of Biomedical Research (IIB Sant Pau), Barcelona, Spain; ${ }^{5}$ Department of Internal Medicine, American University of Beirut, Beirut, Lebanon; ${ }^{6}$ German Cochrane Center, Institute of Medical Biometry and Medical Informatics, University of Freiburg, Germany

\section{0:1136/bmjas-2013-002293.115}

Background Traditional clinical decision support systems in Electronic Medical Records (EMR) use algorithms with inclusion/ exclusion criteria to provide direction to clinicians. Improved systems for developing trustworthy guidelines (e.g. GRADE,) typically include many weak recommendations unsuited for clear inclusion/exclusion criteria, and in which the right decision varies from patient to patient. Through PLUGGED-IN phase 1 we developed a conceptual framework and a guideline authoring/publication platform to allow use of trustworthy guidelines directly as decision support in EMRs, not dependent on traditional algorithms or reproduction of the content. Our framework is based on a multilayered guideline presentation format developed in DECIDE.
Objectives To implement and test our novel approach to decision support where relevant patient specific information is shown alongside evidence based recommendations in EMRs.

Methods We used a web guideline published through the MAGIC (Making Grade the Irresistible Choice) application, which allowed our EMR partner to make use of its structured content, ontology-coded clinical questions and recommendationspecific EMR elements.

Results The EMR system was able to interact with the guideline, suggest relevant recommendations displayed along with relevant patient specific information (lab tests, measurements, medications), and offer these to facilitate direct ordering. We will show real examples and live products.

Discussion Results suggest we can offer a complementary approach to traditional algorithm-based systems that is compatible with a large number of EMRs.

Implications for Guideline Developers/Users PLUGGED-IN provides a model for direct use of guidelines as decision support in EMRs.

\section{IMPLEMENTING A KNOWLEDGE APPLICATION PROGRAM FOR ANXIETY AND DEPRESSION IN COMMUNITY-BASED PRIMARY MENTAL HEALTH CARE: THE CLINICAL DECISION SUPPORT COMPONENT}

${ }^{1,2}{ }^{2}$ Roberge, ${ }^{2,3} \mathrm{~L}$ Fournier, ${ }^{2} \mathrm{H}$ Brouillet, ${ }^{1} \mathrm{~A}$ Benoit. ${ }^{1}$ Faculty of Medicine and Health Sciences, University of Sherbrooke, Sherbrooke, Canada; ${ }^{2}$ Institut National de Santé Publique du Québec, Montreal, Canada; ${ }^{3}$ CRCHUM, University of Montreal, Montreal, Canada

\section{0:1136/bmjqs-2013-002293.116}

Background We developed a knowledge application programme to support the improvement of the organisation and delivery of care for anxiety and depressive disorders in communitybased primary mental health care teams (CMHTs) in Quebec (Canada). The programme is based on the Chronic Care Model, including a Decision Support component, and the PARiHS framework.

Objectives 1- To implement and evaluate a knowledge application programme, 2- to explore barriers and facilitators associated with the implementation of Decision Support strategies, particularly the uptake of clinical practice guidelines.

Methods The design is a mixed-methods prospective multiple case study, with data drawn from the two phases of the project (2008-2010; 2011-2014). Multidisciplinary local working committees in the six CMHTs were required to develop and implement local quality improvement plans with the support of a knowledge broker.

Results While we observed barriers and facilitators at the clinician level in terms of knowledge (e.g familiarity) and attitudes (e.g. applicability, agreement), contextual factors (e.g resources, access) also played an important role in the uptake of clinical practice guidelines into the six local CHMTs.

Discussion Decision Support is a central component of the Chronic Care Model that aims to promote clinical care that is consistent with scientific evidence.

Implications The uptake of evidence in primary care is a complex process that requires careful consideration of the context in which innovations are introduced, and our assessment of barriers and facilitators can be relevant to other primary health care organisations seeking to increase the uptake of anxiety and depression clinical guidelines. 